\title{
Borderline personality disorder symptomatology and age: an analysis using SPECT imaging.
}

\author{
Michelle Blose* \\ Nova Southeastern University, USA
}

Accepted on March 202018

\section{Introduction}

Neuroimaging techniques provide measures of brain structure and function as well as cerebral activity during cognitive processes. These techniques have evaluated the effects of psychiatric illnesses on the brain, specifically, studying how brain-behavior relationships change due to a mental illness. Neuroimaging not only serves as a way to measure the brain structure and function, but it also provides a way to compare neural systems and see how they change due to the progression and treatment of a mental illness. Additionally, neuroimaging facilitates the diagnosis of psychiatric disorders as well as the identification of therapeutic targets [1]. Since the 1970's, neuroimaging has been used to investigate various mental disorders such as schizophrenia and depression [2]. Structural and functional are the two main types of neuroimaging tests. Structural imaging such as computed tomography (CT) and magnetic resonance imaging (MRI) creates an image of the brain's structure including bone, tissue, and blood vessels. Functional imaging such as positron emission tomography (PET), functional magnetic resonance imaging (fMRI) and single photon emission computer tomography (SPECT) reveals activity by measuring blood flow, chemical activity, and electrical impulses [3]. Functional imaging is based on a consistent relationship between regional changes in the cellular activity of the brain and changes in the circulation and metabolism of that region [4]. Since the late 1800's, scientists have known that local blood flow in the brain changes in parallel with changes in cellular activity [4]. Furthermore, brain scans not only show damage to brain tissue, the skull, or blood vessels, but they also assist researchers in studying the effects of healthy brain development, the effects of mental illness, and the effects of mental health treatment on the brain [3]. Thus, neuroimaging is being increasingly applied to the development of psychiatric therapies.

\section{SPECT}

SPECT evaluates regional cerebral blood flow and metabolic activity by using radioisotopes bound to neurospecific pharmaceuticals [5]. SPECT scans develop a two- or threedimensional image using radioactive substance and a special camera. A healthy SPECT scan displays full, even, symmetrical perfusion, with the most intense perfusion in the cerebellum and in the occipital lobes. SPECT imaging is used to assess cerebral perfusion in order to evaluate brain function in, but not limited to the following conditions: impulsivity, bipolar disorder, anxiety disorders, obsessive compulsive disorder, and personality disorders. Contrary to CT and MRI scans, SPECT imaging displays how the brain functions; the blood flow and cell function within the brain allows for a deeper understanding of the complex functions of mental disorders. Similar to PET, it uses a radioactive tracer material and detects gamma rays; however, SPECT emits gamma radiation that is measured directly. According to Little et al. [6], SPECT has been indicated to have less image quality as compared to PET. Along with this, PET may offer increased accuracy and improved sensitivity compared to SPECT scans [7]. Regardless of its limitations, SPECT helps to subtype psychiatric illnesses, helps to increase compliance as patients have visible proof of a problem they may have, and helps family members and patients understand that their mental illness is a medical condition. Also, the American College of Radiology (2015) has indicated that the use of neurological imaging for clinical practice, specifically, SPECT imaging, is beneficial for the evaluation of neurological conditions. Moreover, SPECT imaging is a valuable complementary tool for assisting psychiatric diagnosis, but should be used in conjunction with the classification of psychiatric disorders as set forth in the Diagnostic Statistical Manual of Mental Disorders (DSM) or the International Classification of Diseases (ICD).

\section{Borderline Personality Disorder}

In a given year, about $1.6 \%$ of adults in the United States of American have borderline personality disorder (BPD) [8]. It is researched that the prevalence of BPD may decrease in older groups and is diagnosed predominantly in females, approximately $75 \%$. In the DSM-IV [9], BPD falls under Cluster B Personality Disorders with antisocial personality disorder, histrionic personality disorder, and narcissistic personality disorder. Genetically, as compared to the general population, BPD is five times more common among firstdegree biological relatives. Typically, symptoms of BPD appear in adolescence or early adulthood and, then, individuals typically gain greater stability around ages 30 and 40; however, symptoms such as impulsivity, intensity in relationships, and tendency toward intense emotions are often experienced lifelong. Females are more likely to have comorbid diagnoses of major depression, anxiety disorders, or eating disorders, and males are more likely to have comorbid diagnoses of substance abuse [10]. According to the National Institute of Mental Health-funded National Comorbidity Survey Replication, approximately $85 \%$ of people with BPD meet diagnostic criteria for another mental illness.

\section{Symptom Characteristics}

BPD is marked by unstable moods, behavior and relationships; people who have this disorder most often experience unstable interpersonal relationships with others, impulsive behaviors as 
Citation: Blose M. Borderline personality disorder symptomatology and age: an analysis using SPECT imaging. J Ment Health Aging 2018;2(1):33-35.

well as reckless behaviors and difficulties with regulating emotions and thoughts [10]. BPD is characterized by the following criteria: 1 . frantic efforts to avoid real or imagined abandonment, 2. patterns of unstable and intense interpersonal relationships characterized by alternating between extremes of idealization and devaluation, 3 . identity disturbance: markedly and persistently unstable self-image or sense of self, 4 . impulsivity that is potentially self-damaging, 5. recurrent suicidal behavior, gestures or threats, or self-mutilating behavior, 6. affective instability due to marked reactivity of mood, 7. chronic feelings of emptiness, 8. inappropriate, intense anger or difficulty controlling anger, and 9. transient, stress-related paranoid ideation or severe dissociative symptoms [11]. These symptoms cause the individual significant amounts of distress that negatively impact their quality of life. In addition, seemingly mundane events may trigger symptoms. Currently, Cognitive Behavioral Therapy, Dialectical Behavior Therapy, and Schema-Focused Therapy have been researched to treat BPD. Also, at this time, no medications have been approved by the United States Food and Drug Administration to treat BPD; however, many individuals with BPD will take medication to reduce symptoms of anxiety, depression, or aggression [10]. Therefore, BPD is regarded as a product of complex interactions using genetic, neurochemical, neuroanatomical, and psychological factors.

\section{Neuropsychological Testing and BPD}

Because individuals diagnosed with BPD have shown abnormal affect regulation, weak impulse control, disturbed as well as chaotic interpersonal relationships, and pathological self-image, they have been researched to show neuropsychological impairments [12]. Dysfunctions in response-inhibitory processes, visuomotor skills, verbal memory, decision-making, attention, and visual memory have been found to occur more frequently [13]. Along with these findings, more than $80 \%$ of individuals with BPD have been found to have neuropsychological impairments linked to the dorsolateral prefrontal and orbitofrontal regions. Furthermore, response inhibition has been found to interfere with tasks requiring attention and memory in individuals with BPD. Neuropsychological performance of patients with BPD has been investigated using the Repeatable Assessment of Neuropsychological Status (RBANS) to measure attention, memory as well as visuospatial and language functions. Seres et al. [14] studied 50 borderline patients, 30 patients with other personality disorders (e.g. schizoid, narcissistic, antisocial, histrionic) and 30 healthy controls. Their results revealed a significant neuropsychological impairment in the BPD group compared to the control group as well as BPD group compared to the other personality disorders group; however, the differences were less pronounced when comparing the BPD group and the other personality disorder groups. Post-hoc Tukey tests between the BPD group and the control group revealed differences in three domains: attention, immediate memory, and delayed memory. Along with these findings, visuospatial and language indices did not reveal significant differences. Moreover, results suggest that attention, immediate memory, and delayed memory are the most impaired neurological domains in BPD, which may indicate frontal and temporal lobe dysfunction [14].

\section{In BPD}

Differences in volume and activity in brain structures related to emotion and impulsivity have been investigated in individuals with BPD. BPD has been linked to amygdala and limbic systems of the brain, which control emotions, such as rage, fear and impulsive automatic reactions [15].

\section{PET Scans and BPD}

PET scans have investigated how BPD affects neural activity. Generally, these scans have revealed that people with BPD have showed hypometabolism of glucose in the prefrontal cortex and limbic system [15]. In addition, PET studies have suggested that BPD patients have abnormalities in their frontal and temporal lobes and patients with BPD have shown low glucose metabolism in their frontal cortex (dorsolateral frontal cortex) and limbic system (anterior cingulate cortex) as well as the basal ganglia and thalamus during resting state in 10 patients between the ages of 24 and 45 years diagnosed with BPD [16]. Along with these findings, PET scans observed increased activation of the bilateral dorsolateral prefrontal cortex and right cuneus and decreased activation in the medial prefrontal cortex and various structures involved in visual processing (e.g. left fusiform gyrus, left visual association cortex). Irle et al. [17] examined whether brain glucose metabolism is reduced in temporo-parietal cortices as well as investigated whether temporo-parietal metabolic changes are related to clinical symptoms and to memory deficits of BPD subjects in 17 female inpatients who were experiencing strong dissociative symptoms and memory deficits. In this study, results suggested that there was a reduction in glucose metabolism in right-sided ventromedial temporal and left-sided medial parietal/posterior cingulate cortices [17]. In ventromedial and lateral temporal cortices, memory performance was correlated with metabolic activity whereas poorer memory performance was correlated to lower metabolism.

Furthermore, overall, glucose metabolism is reduced in the prefrontal cortex and temporal lobe.

\section{MRI Scans and BPD}

Orbitofrontal cortex dysfunction with distinct symptom clusters, such as impulsivity, has been associated with BPD. Sixteen women with BPD according to DSM-IV criteria without comorbid posttraumatic stress disorder and 16 healthy females were investigated at resting-state conditions using a MRI. Results revealed that individuals diagnosed with BPD exhibited decreased blood perfusion in the left and right lateral orbitofrontal cortex. Additionally, a positive relationship between medial and lateral orbitofrontal blood flow and impulsivity scores was shown using a correlation analysis [18]. Unlike impulsivity scores, measures of dissociation tension and depression and orbitofrontal cortex blood perfusion did not reveal significant correlations. Furthermore, a measure of 
impulsivity has been shown to relate to blood flow in BPD at resting state in the orbitofrontal cortex.

Brambilla et al. [19] conducted a study that included 10 participants with BPD who had various comorbidities. Their results indicated that individuals with BPD have decreased hippocampus volumes, and individuals who were abused as a child, showed a greater decrease. Also, they found that BPD is associated with an enlarged putamen, particularly in those with comorbid substance use disorders [19].

Another study examining the anatomical changes in BPD using a MRI found that the right parietal cortex was reduced in size and that the hippocampus volume was decreased [17]. This analysis consisted of 30 female patients diagnosed with BPD who had comorbidities of posttraumatic stress disorder, panic disorder, generalized anxiety disorder, major depression, obsessive-compulsive disorder, somatization disorder, anorexia, depersonalization disorder, dissociative amnesia, and dissociative identity disorder. When examining 25 subjects diagnosed with BPD who had no comorbid diagnoses from inpatient and outpatient hospitals, results found that these individuals had reduced frontal lobe volumes by $6.2 \%$. No other significant differences existed when comparing the BPD group to a healthy group [20]. Moreover, MRI scans have shown differences in neural activity, specifically, in the frontal and temporal lobes.

\section{SPECT Scans and BPD}

Reduced regional cerebral blood perfusion in the right lateral temporal cortex and the temporal pole as well as the ventrolateral parts of the right prefrontal cortex have been found in 27 patients with BPD and 10 patients with antisocial personality disorder; all patients were included in the study for impulsive behavior [21]. In summary, the study investigated how BPD and antisocial personality disorder show unusual patterns of blood perfusion using SPECT.

\section{References}

1. Masdue JC. Neuroimaging in psychiatric disorders. Neurotherapeutics. 2011; 8(1):93-102.

2. Zipursky RB, Meyer JH, Verhoeff NP. PET and SPECT imaging in psychiatric disorders. Can J Psychiatry. 2007;52(3):146-57.

3. National Institute of Mental Health. Neuroimaging and mental illness: A window into the brain. 2010.

4. Raichle ME. Functional brain imaging and human brain function. J Neurosci. 2003;23(10):3959-62.

5. Amen D, Willeumier K. Brain SPECT imaging: A powerful, evidence-based tool for transforming clinical psychiatric practice. Minerva Psichiatrica. 2011;52(3): 109-23.

6. Little DM, Arciniegas DB, Hart J. Advanced neuroimaging. In Arciniegas, D.B., Anderson, C.A., \& Filley, C.M. (Eds.), Behavioral neurology and neuropsychiatry. New York: Cambridge University Press. 2013;430-41.
7. Segall G. Assessment of myocardial viability by positron emission tomography. Nuclear Medicine Communications. 2002;23(4):323-30.

8. Lensenweger ME, Lane MC, Loranger AW, et al. DSM-IV personality disorders in the National Comorbidity Survey Replication. Biol Psychiatry. 2007;62(6):552-64.

9. American Psychiatric Association. Diagnostic and statistical manual of mental disorders (5th ed.) Washington, DC: Author. 2014.

10. National Institute of Mental Health. Borderline personality disorder. 2015.

11. American Psychiatric Association. Diagnostic and statistical manual of mental disorders (4th ed., text rev.). Washington, DC: Author. 2000.

12. O'Leary KM. Neuropsychological testing results. Psychiatr Clin N Am. 2000;33(1):41-60.

13. LeGris J, van Reekum R. The neuropsychological correlates of borderline personality disorder and suicidal behaviour. Can J Psychiatry. 2006;51(3):131-42.

14. Seres I, Unoka Z, Bodi N, et al. The neuropsychology of borderline personality disorder: Relationship with clinical dimensions and comparison with other personality disorders. J Pers Disord. 2009;23(6):555-62.

15. Lis E, Greenfield B, Henry M, et al. Neuroimaging and genetics of borderline personality disorder: A review. J Psychiatry Neurosci. 2007;32(3):162-73.

16. De la Fuente JM, Goldman S, Stanus E, et al. Brain glucose metabolism in borderline personality disorder. J Psychiatr Res. 1997;31(5):531-41.

17. Irle E, Lange C, Sachsse U. Reduced size and abnormal asymmetry of parietal cortex in women with borderline personality disorder. Biol Psychiatry. 2005;57(2):173-82.

18. Wolf RC, Thomann PA, Sambataro F, et al. Orbitofrontal cortex and impulsivity in borderline personality disorder: An MRI study of baseline brain perfusion. Eur Arch Psychiatry Clin Neurosci. 2012;262(8), 677-85.

19. Brambilla P, Soloff PH, Sala M, et al. Anatomical MRI study of borderline personality disorder patients. Psychiatry Res. 2004;131(2):125-33.

20. Lyoo IK, Han MH, Cho DY. A brain MRI study in subjects with borderline personality disorder. J Affect Disord. 1998;50(2-3):235-44.

21. Goethals I, Audenaert K, Jacobs F, et al. Brain perfusion SPECT in impulsivity-related personality disorders. Behav Brain Res. 2005;157(1):187-92.

\section{*Correspondence to:}

Michelle Blose

Nova Southeastern University

USA

Tel: +1 800-541-6682

E-mail: michelleannblose@gmail.com 